\title{
A DESREGULAÇÃO DE PROGESTERONA E SEU EFEITO NOS RECEPTORES GABA-A NO TRANSTORNO DISFÓRICO PRÉ-MENSTRUAL
}

\section{PROGESTERONE DEREGULATION AND ITS EFFECT ON GABA-A RECEPTORS IN PREMENSTRUAL DYSPHORIC DISORDER}

\author{
Mariana Oliveira Mourão ${ }^{1 *}$, Elaine de Oliveira Zanini² \\ ${ }^{1}$ Acadêmica do curso de Medicina do Centro Universitário FAG. ${ }^{2}$ Mestre em Desenvolvimento \\ Regional e Agronegócio pela Universidade Estadual do Oeste do Paraná UNIOESTE. \\ *Autor correspondente. E-mail: mari.mouraoo@gmail.com
}

DOI: $10.35984 / f j h . v 2 i 2.169$

\section{RESUMO}

O transtorno disfórico pré-menstrual (TDPM) é um transtorno psiquiátrico que envolve os hormônios ovarianos que acomete o ciclo GABAérgico do sistema nervoso central da mulher. A partir de um estudo qualitativo, foi levantada uma pesquisa sobre o funcionamento fisiopatológico da doença. Os principais aspectos desenvolvidos foram a ação bimodal da alopregnanolona, a hipótese de tolerância dos receptores $\mathrm{GABA}_{\mathrm{A}}$ e 0 uso de progesterona como tratamento da doença. Os resultados da pesquisa indicam que a suplementação de progesterona contribui de maneira agravante à doença, aumentando os sintomas negativos e sendo contraindicada para o tratamento do TDPM.

Palavras-chave: GABA, Progesterona, Transtorno disfórico pré-menstrual.

\begin{abstract}
Premenstrual dysphoric disorder (PMDD) is a psychiatric disorder involving ovarian hormones that affects the GABAergic cycle of a woman's central nervous system. From a qualitative study, a research on the pathophysiological functioning of the disease was raised. The main aspects involved were a bimodal action of allopregnanolone, a hypothesis of tolerance of GABAA receptors and progesterone use as treatment of the disease. Research results indicate that progesterone supplementation exacerbates disease, symptom symptoms and is contraindicated for the treatment of $P M D D$.
\end{abstract}

Keywords: GABA, Progesterone, Premenstrual dysphoric disorder.

\section{INTRODUÇÃO}

O Transtorno Disfórico Pré-Menstrual (TDPM) é um transtorno psiquiátrico presente em 2 a $10 \%$ de toda a população feminina em idade reprodutiva, sendo caracterizado por uma intensificação e agravamento dos sintomas do Transtorno PréMenstrual (TPM) durante a fase lútea do ciclo menstrual (BATHIA e BATHIA, 2002). Sua etiologia não é completamente compreendida, no entanto, estudos sugerem o envolvimento de hormônios esteróides ovarianos (WANG et al, 1996) e da função GABAérgica (SUNDSTRÖM-POROMAA, SMITH e GULINELLO, 2003) na doença.

É de extrema importância saber diferenciar o TDPM do TPM, que acomete $80 \%$ de mulheres (VALADARES et al, 2006), e dos sintomas apresentados por outras doenças psiquiátricas e clínicas pois, dessa maneira, é incrementada a qualidade do diagnóstico e do tratamento ofertados às mulheres portadoras da doença. 
De acordo com a 5a edição do Manual Diagnóstico e Estatístico de Transtornos Mentais - DSM-5, publicado em 2014 pela Associação Americana de Psiquiatria, "as características essenciais do transtorno disfórico pré-menstrual são a expressão de labilidade do humor, irritabilidade, disforia e sintomas de ansiedade que ocorrem repetidamente durante a fase pré-menstrual do ciclo e remitem por volta do início da menstruação ou logo depois". Tais sintomas "devem estar associados a sofrimento clinicamente significativo e/ou prejuízo claro e acentuado na capacidade de funcionar social e profissionalmente", além de serem observados por, no mínimo, 2 meses.

Ainda de acordo com o DSM-5, "os sintomas atingem seu auge perto do momento de início da menstruação. Embora não seja incomum perdurarem até os primeiros dias da menstruação, a mulher deve ter um período livre de sintomas na fase folicular depois que inicia o período menstrual".

Normalmente, durante a fase lútea de uma mulher saudável, são notados níveis crescentes do hormônio esteróide progesterona. Um dos metabólitos desse hormônio, a alopregnanolona $\left(\mathrm{C}_{21} \mathrm{H}_{34} \mathrm{O}_{2}\right)$, age como um modulador positivo de receptores $\mathrm{GABA} A$ no cérebro, estando relacionada à ativação de seus canais iônico (MAJEWSKA et al, 1986). Esses receptores estão associados com o ácido gama-aminobutírico (GABA), considerado o principal neurotransmissor inibitório no córtex cerebral (MCCORMICK, 1989) já que, uma vez ativado, ocorre influxo de íons cloreto responsáveis por hiperpolarizar a membrana pós-sináptica, ou seja, torna a célula seguinte à transmissão sináptica menos propensa a ser excitada (SIEGHART, 1995). Dessa maneira, é possível dizer que o hormônio esteróide está envolvido no controle do humor e do comportamento das mulheres, seja de forma direta ou indireta.

Entretanto, dentro da patologia do TDPM a mulher possui níveis reduzidos de GABA em sua concentração plasmática durante sua fase lútea, sendo isso consequência dos observados declínios acentuados de progesterona e, portanto, de alopregnanolona durante a citada fase do ciclo menstrual (KAUR, GONSALVES e THACKER, 2004). Dessa forma, é justificada a presença dos sintomas negativos, tais como ansiedade, impulsividade, raiva e labilidade afetiva (DUCASSE et al, 2016).

Em um transtorno depressivo (atual classificação do TDPM, de acordo com o DSM-5), assim como em outros transtornos de humor, os níveis de GABA são menores em comparação aos observados em pessoas saudáveis (SANACORA et al, 1999). Esses níveis reduzidos podem ter como causa disfunções relacionadas à síntese de GABA ou às enzimas envolvidas no ciclo GABAérgico (GUERRA-ARAIZA et al, 2008). Tendo em vista o dado anterior juntamente aos atuais tipos de tratamento, o uso de antidepressivos da classe inibidores seletivos da recaptação de serotonina (ISRS) são considerados a linha de tratamento número um para o TDPM, tendo apresentado maior eficácia em tratamentos contínuos em comparação a tratamentos de uso desregulado (SHAH et al, 2008). Os neurotransmissores serotoninérgicos têm ação direta em neurônios gabaérgicos presentes no córtex cerebral (SMILEY e GOLDMAN-RAKIC, 1996), uma vez que neles estão expostos receptores 5-HT3 ou 5HT2A para a serotonina (5-HT) (JAKAB e GOLDMAN-RAKIC, 2000), modulando e aumentando os níveis de liberação de GABA (ABI-SAAB et al, 1999).

Esse estudo teve como intuito expor os efeitos da desregulação da progesterona no organismo de mulheres, correlacionando-a às baixas concentrações plasmáticas de GABA, a fim de apoiar a hipótese de que tal anormalidade contribui de forma significativamente negativa para a patogênese da doença. 


\section{METODOLOGIA}

A partir de uma análise qualitativa, foi feita uma revisão de literatura com base nos bancos de dados MEDLINE e PUBMED. Foram encontrados 30 artigos científicos e publicações periódicas entre os anos de 1986 e 2016 que envolvem informações sobre o TDPM e aspectos relacionados à patologia e à fisiologia da doença. Além disso, informações também foram coletadas em livros voltados para a área da Medicina.

\section{RESULTADOS E DISCUSSÃO}

A alopregnanolona, assim como os benzodiazepínicos, os barbitúricos e o álcool, possui ação bimodal frente aos sintomas de humor. Estudos realizados trouxeram resultados de que, em altas doses, o metabólito em questão tem efeitos como ação anticonvulsivante (LANDGREN e SELSTAM, 1995), anestésica (CARL et al, 1990) e hipnótica (SUNDSTROM et al, 1998), enquanto em baixas doses pode estar relacionada à agressividade (MICZEK, WEERTS e DEBOLD, 1993), à irritabilidade (YOSHIMURA e OGAWA, 1989), à dificuldades de memória e de aprendizado (JOHANSSON et al, 2002) e ao humor depressivo (WENZEL et al, 2002). No entanto, dentro da patologia do TDPM esses achados são controversos.

Há hipóteses de que, diferente das mulheres saudáveis, as mulheres com TDPM, apresentam em seus receptores $\mathrm{GABA}_{\mathrm{A}}$ localizados no cérebro alta tolerância frente aos níveis de alopregnanolona, ou seja, sua sensibilidade é reduzida (SUNDSTRÖM et al, 1998; NYBERG et al, 2004). As respostas celulares são comprometidas ao ponto de não ser observada mudança significativa que demonstre a ativação desses receptores quando em contato com a alopregnanolona. Isso está de acordo com a sensibilidade restringida apresentada pelas mulheres com TDPM quando em contato com substâncias que possuem propriedades benzodiazepínicas (SUNDSTRÖM, NYBERG e BÄCKSTRÖM, 1997). Como demonstrou Kask et al (2008), a tolerância dos receptores $\mathrm{GABA}_{A}$ evidenciou também o aumento da resposta de sobressalto (resposta musculoesquelética súbita em resposta a estímulos inócuos: normalmente barulho ou som alto), podendo contribuir para o aparecimento dos sintomas do TDPM.

Foram realizados experimentos laboratoriais em ratos fêmeas com o intuito de observar os efeitos comportamentais e farmacológicos do tratamento com suplementação hormonal de progesterona. Foi constatado que, com o aumento da expressão da alopregnanolona, houve maior excitabilidade neuronal (GULINELLO et al, 2001). Dessa maneira, mudanças de comportamentos tendendo, principalmente, para o comportamento do tipo ansioso foram observadas pelo mesmo autor. Em outros estudos realizados na mesma época, foram descritos o mesmo padrão comportamental. No ano de 2000, Beauchamp et al observou que os ratos submetidos ao mesmo experimento apresentaram aversão ao ambiente em que estes estavam inseridos, denotando, assim, atitudes típicas de um comportamento ansioso.

Experimentos com o mesmo intuito também foram realizados em pleno organismo humano. Em 2005, um estudo por Andréen et al teve como base a aplicação randomizada, por meio de supositórios vaginais, de doses baixas, médias e altas de progesterona, placebo e estrogênio em mulheres que já haviam passado pela menopausa. As que receberam as doses de progesterona foram, consequentemente, acometidas com maior concentração plasmática de alopregnanolona. Ao compará-las com as mulheres que receberam doses de outras substâncias, elas apresentaram um FAG Journal of Health - ISSN 2674-550X, 2020, v.2, n.2, p. 295 
aumento significativo de sintomas negativos. Esse mesmo resultado foi visto no estudos realizados por Schmidt et al (1998) e por Segebladh et al (2009), em que cada qual descreve que a dose mais alta da combinação de estradiol e progesterona administrada fora associada à recorrência mais acentuada dos sintomas da doença.

\section{CONSIDERAÇÕES FINAIS}

Com base no estudo qualitativo realizado sobre a progesterona dentro da fisiopatologia do TDPM, os ensaios revisados sugerem que o hormônio esteróide em questão representa um risco consideravelmente elevado quando visto sob a perspectiva de ser um aliado ao tratamento da doença. Sugere-se que novas pesquisas e ensaios sejam realizados para que se saiba mais sobre o envolvimento dos hormônios esteróides e seus derivados no TDPM, visto que esses possuem grande potencial contributivo para a elaboração de ações profiláticas e de tratamentos a partir do conhecimento de seus funcionamentos fisiológicos dentro da patologia.

\section{REFERÊNCIAS}

ABI-SAAB, W.M.; BUBSER, M.; ROTH, R.H.; DEUTCH, A.Y. 5-HT2 Receptor Regulation of Extracellular GABA Levels in the Prefrontal Cortex. $\begin{array}{lllllll}\text { Neuropsychopharmacology, } & \text { v. } 20, \quad \text { n. } 1, \quad \text { p. } & 92-96, & \end{array}$ https://doi.org/10.1016/S0893-133X(98)00046-3

ANDRÉEN, L.; SUNDSTRÖM-POROMAA, I.; BIXO, M.; ANDERSSON, M.; NYBERG, S.; BÄCKSTRÖM, T. Relationship Between Allopregnanolone and Negative Mood in Postmenopausal Women Taking Sequential Hormone Replacement Therapy with Vaginal Progesterone. Psychoneuroendocrinology, v. 30, n. 2, p. 212-224, 2005. https://doi.org/10.1016/j.psyneuen.2004.07.003

ASSOCIAÇÃO AMERICANA DE PSIQUIATRIA. Manual Diagnóstico e Estatístico de Transtornos Mentais - DSM-5, 5a․ edição. Washington DC: AMERICAN PSYCHIATRIC ASSOCIATION PRESS, 2014.

BEAUCHAMP, M.H.; ORMEROD, B.K.; JHAMANDAS, K.; BOEGMAN, R.J.; BENINGER, R.J. Neurosteroids and Reward: Allopregnanolone Produces a Conditioned Place Aversion in Rats. Pharmacology Biochemistry and Behavior, v. 67, n.1, p. 29-35, 2000. https://doi.org/10.1016/s0091-3057(00)00299-9

BHATIA, S. C.; BHATIA, S. K. Diagnosis and Treatment of Premenstrual Dysphoric Disorder. American Family Physician, v. 66, n. 7, p. 1239-1248, 2002.

CARL, P.; HÖGSKILDE, S.; NIELSEN, J.W.; SÖRENSEN, M.B.; LINDHOLM, M.; KARLEN, B.; BÄCKSTRÖM, T. Pregnanolone Emulsion: A Preliminary Pharmacokinetic and Pharmacodynamic Study of a New Intravenous Anaesthetic Agent. Anaesthesia, v. 45, n. 3, p. 189-197, 1990. https://doi.org/10.1111/i.13652044.1990.tb14683.x

DUCASSE, D.; JAUSSENT, I.; OLIÉ, E.; GUILLAUME, S.; LOPEZ-CASTROMAN, J.; COURTET, P. Personality Traits of Suicidality Are Associated with Premenstrual 
Syndrome and Premenstrual Dysphoric Disorder in a Suicidal Women Sample. PLOS ONE, v. 11, n. 2, e0148653, 2016. https://doi.org/10.1371/journal.pone.0148653

GUERRA-ARAIZA, C.; MIRANDA-MARTINEZ, A.; NERI-GOMÉZ, T.; CAMACHOARROYO, I. Sex Steroids Effects on the Content of GAD, TH, GABAA, and Glutamate Receptors in the Olfactory Bulb of the Male Rat. Neurochemical Research, v. 33, n. 8, p. 1568-1573, 2008. https://doi.org/10.1007/s11064-008-9665-1

GULINELLO, M.; GONG, Q.H.; LI, X.; SMITH, S.S. Short-term Exposure to a Neuroactive Steroid Increases a4 GABAA Receptor Subunit Levels in Association with Increased Anxiety in the Female Rat. Brain Research, v. 910, n. 0, p. 55-66, 2001.

JAKAB, R.L.; GOLDMAN-RAKIC, P.S. Segregation of Serotonin 5-HT2A and 5-HT3 Receptors in Inhibitory Circuits of the Primate Cerebral Cortex. The Journal of Comparative Neurology, v. 417, n. 3, p. 337-348, 2000. https://doi.org/10.1002/(sici)1096-9861(20000214)417:3\%3C337::aidcne7\%3E3.0.co;2-0

JOHANSSON, I.M.; BIRZNIECE, V.; LINDBLAD, C.; OLSSON, T.; BÄCKSTRÖM, T. Allopregnanolone Inhibits Learning in the Morris Water Maze. Brain Research, v. 934, n. 2, p. 125-131, 2002. https://doi.org/10.1016/s0006-8993(02)02414-9

KASK, K.; GULINELLO, M.; BÄCKSTRÖM, T.; GEYER, M.A.; SUNDSTRÖMPOROMAA, I. Patients with Premenstrual Dysphoric Disorder Have Increased Startle Response Across Both Cycle Phases and Lower Levels of Prepulse Inhibition During the Late Luteal Phase of the Menstrual Cycle. Neuropsychopharmacology, v. 33, n. 9, p. 2283-2290, 2008. https://doi.org/10.1038/sj.npp.1301599

KAUR, G.; GONSALVES, L.; THACKER, H.L. Premenstrual Dysphoric Disorder: a Review for the Treating Practitioner. Cleveland Clinic Journal of Medicine, v. 71, $\mathrm{n}$. 4, p. 303-305, 2004. https://doi.org/10.3949/ccjm.71.4.303

LANDGREN, S.; SELSTAM, G. Interaction Between 17-beta-oestradiol and 3-alphahydroxy-5-alpha-pregnane-20-one in the Control of Neuronal Excitability in Slices from The CA1 Hippocampus In Vitro of Guinea-pigs and Rats. Acta Physiologica, v. 154, n. 2, p. 165-176, 1995. https://doi.org/10.1111/j.1748-1716.1995.tb09898.x

MAJEWSKA, M. D.; HARRISON, N. L.; SCHWARTZ, R. D.; BARKER, J. L.; PAUL, S. M. Steroid Hormone Metabolites Are Barbiturate-Like Modulators of the GABA Receptor. Science, v. 234, n. 4753, p. 1004-1007, 1986. https://doi.org/10.1126/science.2422758

MCCORMICK, D. A. GABA as an Inhibitory Neurotransmitter in Human Cerebral Cortex. Journal of Neurophysiology, v. 62, n. 5, p. 1018-1027, 1989. https://doi.org/10.1152/jn.1989.62.5.1018

MICZEK, K.A.; WEERTS, E.M.; DEBOLD, J.F. Alcohol, Benzodiazepine-GABAA Receptor Complex and Aggression: Ethological Analysis of Individual Differences in Rodents and Primates. Journal of Studies on Alcohol, v.11, n.11, p. 170-179, 1993. http://dx.doi.org/10.15288/jsas.1993.s11.170 
NYBERG, S.; WAHLSTRÖM, G.; BÄCKSTRÖM, T.; SUNDSTRÖM-POROMAA, I. Altered Sensitivity to Alcohol in the Late Luteal Phase Among Patients with Premenstrual Dysphoric Disorder. Psychoneuroendocrinology, v. 29, n. 6, p. 767777, 2004. https://doi.org/10.1016/S0306-4530(03)00121-5

SANACORA, G.; MASON, G.F.; ROTHMAN, D.L.; BEHAR, K.L.; HYDER, F.; PETROFF, O.A.C.; BERMAN, R.M.; CHARNEY, D.S.; KRYSTAL, J.H. Reduced Cortical Gamma-Aminobutyric Acid Levels in Depressed Patients Determined by Proton Magnetic Resonance Spectroscopy. Archives of General Psychiatry, v. 56, n. 11, p.1043-1047, 1999. https://doi.org/10.1001/archpsyc.56.11.1043

SCHMIDT, P.J.; NIEMAN, L.K.; DANACEAU, M.A.; ADAMS, L.F.; RUBINOW, D.R. Differential Behavioral Effects of Gonadal Steroids in Women With and in Those Without Premenstrual Syndrome. The New England Journal of Medicine, v. 338, n. 4, p. 209-216, 1998. https://doi.org/10.1056/NEJM199801223380401

SEGEBLADH, B.; BORGSTRÖM, A.; NYBERG, S.; BIXO, M.; SUNDSTRÖMPOROMAA, I. Evaluation of Different Add-back Estradiol and Progesterone Treatments to Gonadotropin-releasing Hormone Agonist Treatment in Patients with Premenstrual Dysphoric Disorder. American Journal of Obstetrics and $\begin{array}{llllll}\text { Gynecology, } & \text { v. 201, n. } & \text { 2, p. 139.e1-139.e8, }\end{array}$ https://doi.org/10.1016/j.ajog.2009.03.016

SHAH, N.R.; JONES, J.B.; APERI, J.; SHEMTOV, R.; KARNE, A.; BORENSTEIN, J. Selective Serotonin Reuptake Inhibitors for Premenstrual Syndrome and Premenstrual Dysphoric Disorder: a Meta-analysis. Obstetrics and Gynecology, v. 111, n. 5, p. 1175-1182, 2008. https://doi.org/10.1097/AOG.0b013e31816fd73b

SIEGHART, W. Structure and Pharmacology of y-Aminobutyric Acid A Receptor Subtypes. Pharmacological Reviews, v. 46, n. 2, 181-234, 1995.

SMILEY, J.F.; GOLDMAN-RAKIC, P.S. Serotonergic Axons in Monkey Prefrontal Cerebral Cortex Synapse Predominantly on Interneurons as Demonstrated by Serial Section Electron Microscopy. The Journal of Comparative Neurology, v. 367, n. 3, p. $\quad 431-443, \quad 1996 . \quad$ https://doi.org/10.1002/(SICl)10969861(19960408)367:3\%3C431::AID-CNE8\%3E3.0.CO;2-6

SUNDSTRÖM, I.; ANDERSSON, A.; NYBERG, S.; ASHBROOK, D.; PURDY, R.H.; BÄCKSTRÖM, T. Patients with Premenstrual Syndrome Have a Different Sensitivity to a Neuroactive Steroid During the Menstrual Cycle Compared to Control Subjects. $\begin{array}{lllllll}\text { Neuroendocrinology, } & \text { v. } & 67, & \text { n.2, } & \text { p. } & 126-138, & 1998 .\end{array}$ https://doi.org/10.1159/000054307

SUNDSTRÖM, I.; NYBERG, S.; BÄCKSTRÖM, T. Patients with Premenstrual Syndrome Have Reduced Sensitivity to Midazolam Compared to Control Subjects.

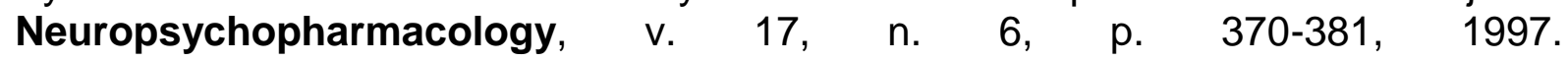
https://doi.org/10.1016/S0893-133X(97)00086-9 
SUNDSTRÖM-POROMAA, I.; SMITH, S.; GULINELLO, M. GABA Receptors, Progesterone and Premenstrual Dysphoric Disorder. Archives of Women's Mental Health, v. 6, n. 1, p. 23-41, 2003. https://doi.org/10.1007/s00737-002-0147-1

VALADARES, G.C.; FERREIRA, L.V.; CORREA FILHO, H.; ROMANO-SILVA, M.C. Transtorno disfórico pré-menstrual revisão - conceito, história, epidemiologia e etiologia. Revista de Psiquiatria Clínica, v. 33, n. 3, p. 117-123, 2006. http://dx.doi.org/10.1590/S0101-60832006000300001

WANG, M.; SEIPPEL, L.; PURDY, R.H.; BÄCKSTRÖM, T. Relationship Between Symptom Severity and Steroid Variation in Women with Premenstrual Syndrome: Study on Serum Pregnenolone, Pregnenolone Sulfate, 5-alpha-pregnane-3,20-dione and 3-alpha-hydroxy-5-alpha-pregnan-20-one. Journal of Clinical Endocrinology $\begin{array}{llllll}\text { and Metabolism, } & \text { v. } 81, \quad \text { n. } 3, \quad \text { p. 1076-1082, } & 1996 .\end{array}$ https://doi.org/10.1210/jcem.81.3.8772579

WENZEL, R.R.; BARTEL, T.; EGGEBRECHT, H.; PHILIPP, T.; ERBEL, R. Centralnervous Side Effects of Midazolam During Transesophageal Echocardiography. Journal of the American Society of Echocardiography, v. 15, n. 10 (pt. 2), p. 12971300, 2002. https://doi.org/10.1067/mje.2002.124572

YOSHIMURA, H.; OGAWA, N. Acute and Chronic Effects of Psychotropic Drugs on Maternal Aggression in Mice. Psychopharmacology (Berl), v. 97, n. 3, p. 339-342, 1989. https://doi.org/10.1007/bf00439447 